\title{
Some lichens from Kashmar, NE Iran
}

\section{Mahroo H. Moniri ${ }^{1}{ }^{*}$, Aazam Soltani ${ }^{1}$ and Saleh Kamyabi ${ }^{1}$}

${ }^{1}$ Biology Department, Faculty of Science, Islamic Azad University, Mashhad Branch, Mashhad, Iran.

* Biology Department, Faculty of Science, Islamic Azad University, Rahnamaie str., Mashhad, Iran

* Corresponding author. E-mail: m.h.moniri@mshdiau.ac.ir

\begin{abstract}
The present paper is based on recent collection of lichens made between 2007-2008 on Kashmar in the central part of the Razavi Khorasan province. A total of 31 taxa of lichens are reported from three localities in the investigated area. Two species are new to Iran, eight species are new to the province.
\end{abstract}

Keywords: Lichenized fungi, Iran, New record

\section{INTRODUCTION}

The current lichen diversity of Iran remained poorly studied until recently (Szatala., 1940, 1957). Iran's first checklist has published in 2004 by Seaward et al. which includes 396 lichenized fungi. Of course in the revised mentioned checklist, 63 species have been recorded from Razavi_Khorasan province so far (Seaward et al., 2008). Although it seems that there is more species in this zone based on the different climate, heights and the substrates. In the course of approach to better result in determination of the lichenized fungi in Razavi Khorasan, Kalate albalou countryside of Kashmar with a surface area of ca. 2/78 $\mathrm{km}^{2}$, in the center of the province has been investigated in this study. Only a few data on lichen biodiversity are available for Kashmar (Hadjmoniry et al., 2005). The area is located between $35^{\circ} 2023122-35^{\circ} 2122922 \mathrm{~N}$ and $58^{\circ} 262722-58^{\circ} 2725922 \mathrm{E}$ (Fig. 1), altitude range from 1380 to $2040 \mathrm{~m}$. At the point of geomorphological, Alkaloid feldspar granite rocks predominated in this area which related to Oligocene period (Aghanabati, 2004). The average annual temperature and humidity are recorded $17 / 6$ and $40 \%$ respectively. The vegetation in the area is steppic plants with Juglans regia L., the dominant wooden species (Rashed et al., 1982-1987).

The present study which is the first taxonomic investigation of the lichen flora of Kalate albalou, has a primary objective: to provide a floristic account of the lichen and increase our knowledge of lichens in the province.

\section{MATERIALS AND METHODS}

During 2007-2008, more than 100 samples were collected from the three localities are listed below in the investigated area by the second author.

1. IRAN: Razavi_Khorasan province, Kashmar, Kalate albalou, $35^{\circ} 2025122 \mathrm{~N}$ and $58^{\circ} 2724522 \mathrm{E}, 1440 \mathrm{~m}$.
2. IRAN: Razavi_Khorasan province, Kashmar, Kalate albalou, $35^{\circ} 2025522 \mathrm{~N}$ and $58^{\circ} 2721522 \mathrm{E}, 1560 \mathrm{~m}$.

3. IRAN: Razavi_Khorasan province, Kashmar, Kalate albalou, $35^{\circ} 2121022 \mathrm{~N}$ and $58^{\circ} 2624022 \mathrm{E}, 1850 \mathrm{~m}$.

Due to the inadequacy of the literature available for identification about 40 samples could be identified to species level so far. The samples were identified by using anatomical and morphological techniques, usual chemical reagents. The study is based on a comprehensive evaluation of the literature mostly Purvis et al. 1992, Nash et al. 2002 and herbarium specimens. The 4-digit numbers indicate voucher specimens deposited in the first author's lichen collection, duplicated in $\mathrm{FUMH}^{+}$with selected specimens in B.

Taxa reported: The Collection is representative of 31 species, 16 genera, 11 families including two new records to Iran $(*)$ and eight to the province $(* *)$.

**Acarospora anatolica H. Magn.

Thallus squamulose, apothecium usually less; disc 0.1$0.25 \mu \mathrm{m}$ diam; hymenium 120-135 $\mu \mathrm{m}$ tall, upper part yellow-brown; paraphyses $0.1-1.5 \mu \mathrm{m}$ thick; colorless; asci more than 200-spored, clavate, 95-110 × 17-20 $\mu \mathrm{m}$; ascospores sometimes ellipsoid 3-5 $\times 2 \mu \mathrm{m}$.

Specimen Examined: 1: on Alkaloid feldspar granite rock, 2007, \# 2043.

Acarospora bullata Anzi

Thallus crustose, pale brown, reddish-brown to dark brown, epruinose, orbicular, distinctly lobate at periphery, marginal lobes 1-2 mm long, $1 \mathrm{~mm}$ wide, at center rimulose-areolate; apothecium sunken in central areoles; ascospores ellipsoid, 3-4(-5) $\times 1.7 \mu \mathrm{m}$.

Specimen Examined: 1: on Alkaloid feldspar granite rock, 2007, \# 2044.

Acarospora strigata (Nyl.) Jatta

Thallus squamulose, cracked-areolate, \pm white or blue

${ }^{+}$Ferdosi University Mashhad Herbarium 


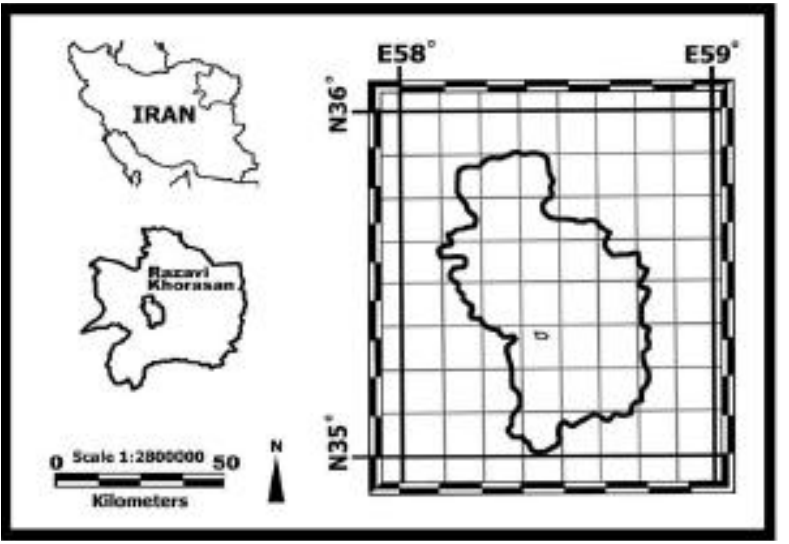

Fig. 1. Geomorphological area of Iran.

pruinose, apothecium scarce, 1-1.5 mm diam, 100-120 (-140) $\mu \mathrm{m}$ high; asci 100-200 spored, ascospores clavate, 3-4.5 ×2-2.5 $\mu \mathrm{m}$.

Specimen Examined:-2: on Alkaloid feldspar granite rock, 2007, \# 2046.

Aspicilia calcarea (L.) Körb.

Thallus crustose, rather thick, continuous or usually cracked-areolate, chalky or grey-white, more infrequently grayish; ascomata apothecia; apothecium \pm immersed, rounded or angular; disc black, not or slightly whitepruinos; asci 4-spored; ascospores 18-30 × 14-27 $\mu \mathrm{m}$, broadly ellipsoid to subglobose, with a thin perispore. Specimen Examined: -3: on Alkaloid feldspar granite rock, 2008, \# 2047.

Aspicilia desertorum (Krempelh.) Mereschk.

Thallus crustose, thick to very thick, to $5 \mathrm{~mm}$ thick, lightto dark-brown, muddy yellow, blackish olive, or rusty red, areolate; apothecium numerous, immersed, 1-4(10) per areole, large, (0.5)1-3.5(5.5-7) mm wide, rounded, older one usually shapeless, often bent; disc black, matt, bare, or finely white-pruinose, concave then flat or with flexuose surface; asci 1-4 spored; ascospores uniseriate, almost globose (31)15-22(28) $\mu \mathrm{m}$.

Specimen Examined: -3: on Alkaloid feldspar granite rock, 2008, \# 2048.

\section{**Aspicilia oxneriana O.B. Blum}

Thallus foliose, monophylous, yellowish-olive, yellowish-brownish, or brownish-yellow above, lower surface of the thallus light brownish-yellow, smooth, sometimes with small pits, mostly in the center; apothecium numerous, 1-4(5) per areole, at first immersed, later emergent; disc black or blackish-brown, densly whitish pruinose, often cracked, rounded, at first concave, later flat to convex; asci cylindrical-clavate, (1)3-4(8)spored; ascospores (16)21-29(31) $\mu \mathrm{m}$, globose.

Specimen Examined: -3: on Alkaloid feldspar granite rock, 2008, \# 2049.

Caloplaca biatorina (A. Massal.) J.Steiner

Thallus crustose, lobed, yellow, orang-red, isidia and

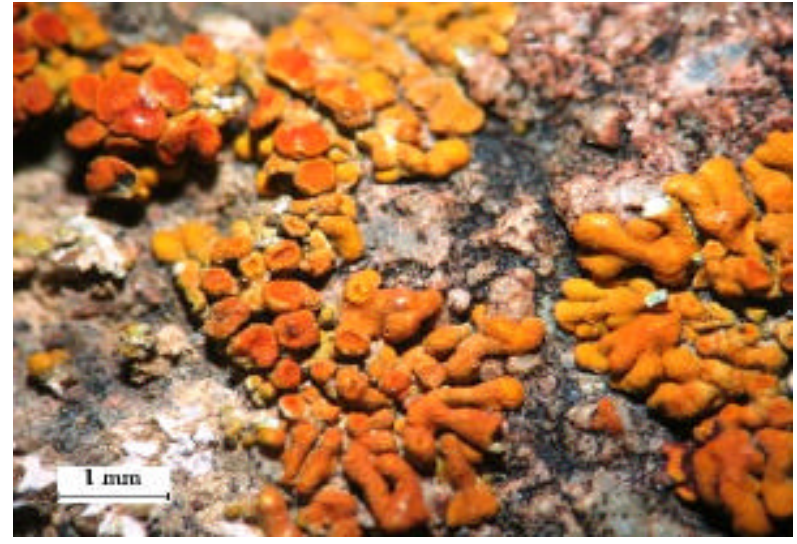

Fig. 2. Thallus of Caloplaca microthalina (Wedd.) Zahlbr.

soredia absent,1-1.5 wide; ascomata apothecia, apothecium lecanorine; paraphyses often swollen; ascospores $9-5 \times 16-9 \mu \mathrm{m}$, ellipsoid.

Specimen Examined:-2: on Alkaloid feldspar granite rock, 2007, \# 2050.

*Caloplaca microthalina (Wedd.) Zahlbr. (Fig. 2)

Thallus of contiguous or often \pm scattered, small, individual microsquamules, yellowish orang; ascomata apothecia; apothecium to $1 \mathrm{~mm}$ diam, yellow-orang, at first, reduced or \pm crenulate when mature; paraphyses often with apical cell swollen; ascospores $12-15 \times 6-8$ $\mu \mathrm{m}$, ellipsoid, septum (1-)3 $\mu \mathrm{m}$ wide.

Specimens Examined: -2: on Alkaloid feldspar granite rock, 2007, \# 2051, 2153.

**Caloplaca variabilis (Pers.) Müll. Arg.

Thallus crustose, gery to deep grey or grayish brown; apothecium black, to $1 \mathrm{~mm}$ diam, scattered to crowded, \pm sessile, flat, becoming slightly convex; epithecium pale; paraphyses broadening and becoming rather stout towards the tips; ascospores $14-16 \quad(-21) \times 7-9 \mu \mathrm{m}$, ellipsoid, septum 2-3(-5) $\mu \mathrm{m}$ wide.

Specimens Examined: -1: on Alkaloid feldspar granite rock, 2007, \# 2052, 2074

\section{Candelariella aurella (Hoffm.) Zahlbr.}

Thallus of scattered, yellow, to green- yellow, convex granules; ascomata apothecia; apothecium discrete, \pm regularly dispersed, yellow; asci 8-spored; ascospores 10-18 × 5-6 $\mu \mathrm{m}$, oblong, ellipsoid, straight or curved. Specimen Examined: -3: on Alkaloid feldspar granite rock, 2008, \# 2053.

* Candelariella medians (Nyl.) A. L. Sm. (Fig. 3)

Thallus placodioid, orbicular, radiating, yellow, citrine or grey-green yellow, the centre granular-areolate to minutely coralloid-isidiat; apothecium small, $0.3-1.2 \mathrm{~mm}$ diam, occasional, dull yellow, flat to slightly convex with a smooth to crenulate margin; asci 8-spored; ascospores 11-17 × 4-6 $\mu \mathrm{m}$, simple, occasionally 1-septate, rather variable, ellipsoid, oblong, tear-drop or slipper-shaped. Specimens Examined: -2: on Alkaloid feldspar granite 


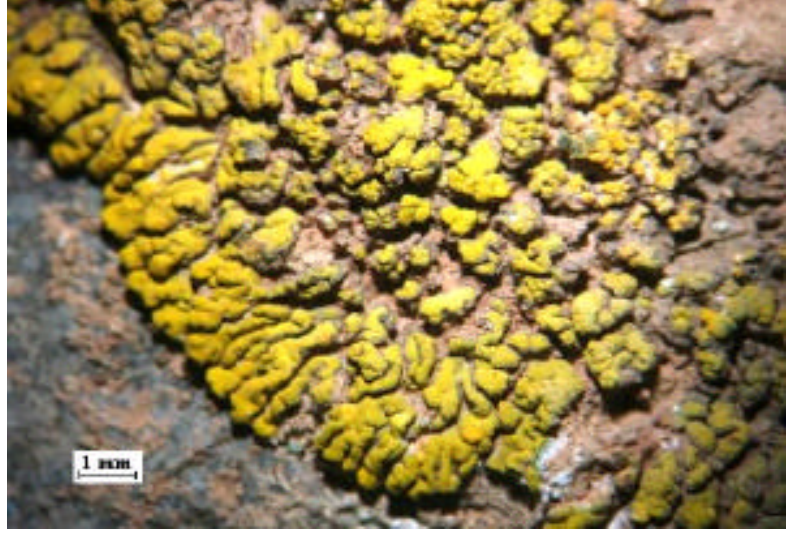

Fig. 3.Thallus of Candelariella medians( Nyl.) A. L. Sm.

rock, 2007, \# 2054, 2256, 2383.

**Candelariella vitellina (Hoffm.) Müll. Arg.

Thallus yellow, orang to brown-orang, continuous and coarsely cracked or in scattered patches, usually rather thick, composed of nodular or squamulose granules; apothecium frequent, $0.5-1.5 \mathrm{~mm}$ diam, flat, margin prominent, persistent, smooth to crenulate; disc grayish yello, sometimes darkening when old; asci (12-)16- to 32spored; ascospores $9-15 \times 3.0-6.5 \mu \mathrm{m}$, simple to weakly 1-septat.

Specimen Examined:-3: on Alkaloid feldspar granite rock, 2008, \# 2055.

\section{Dermatocarpon miniatum (L.) W. Mann}

Thallus foliose, single-lobed and attached to substratum by a usually central holdfast, or multi-lobed; upper surface reddish brown to grey-brown, grey-white pruinose;lower surface pale tan-brown, smooth or warted; ascomata perithecia; asci 50-67 $\times 9-12 \mu \mathrm{m}$, cylindrical; ascospores (8-)9-12(-14) × (4.5)5-6(-7) $\mu \mathrm{m}$, ellipsoid or ovoidellipsoid.

Specimens Examined: -3: on Alkaloid feldspar granite rock, 2008, \# 2056, 2082.

\section{Dimelaena oreina (Ach.) Norman}

Thallus thin to thick, placodioid, with radiate-plicate marginal lobes, areolate towards the center; surface greenish yellow due to usnic acid in the cortex; ascomata apothecia; apothecium black, lecanorine, innate or more usually adnate; disc black or or sometimes whitepruinose, plane to slightly convex; asci cylindrical, 8spored; ascospores brown, 1-septat, 9-13 $\times 5-7 \mu \mathrm{m}$. Specimen Examined: -2: on Alkaloid feldspar granite rock, 2007, \# 2057.

Glypholecia scabra (Pers.) Müll. Arg.

Thallus foliose and umbilicate, white to gray, areolate; apothecium brown, sunken in the thallus; asci more than 8-spored; ascospores simple.

Specimens Examined: -1: on Alkaloid feldspar granite rock, 2007, \# 2058, 2080.

Lecanora crenulata Hook
Thallus usually immersed and inconspicuous, more rarely areolate, pale grey; ascomata apothecia; apothecium constricted at the base slightly raised, often in small groups and then becoming angular by compression; thalline exciple well developed, persistent, white, regularly deeply crenate with 5-8 segments, discs rather variable in colour, reddish brown to yellowish or brownish-black, grey or blue-grey-pruinose; epithecium brownish or blue; hymenium 55-70 $\mu \mathrm{m}$, yellow-brown above; paraphyese sparsely branched, apices capitate, the terminal cell brownish and swollen; ascospores 6$10(-15.5) \times \quad(4-) 4.5-6(-7) \mu \mathrm{m}$.

Specimen Examined:-2: on Alkaloid feldspar granite rock, 2007, \# 2059.

**Lecanora dispersa (L.) Sommerf.

Thallus immersed or sometime consisting of scattered granules, white to pale grey; apothecium sessile, constricted below, thalline exciple well developed, persistent, entire to crenulate to contorted or flexuose; discs very variable in colour, pinkish brown to olivaceous brown, or pale yellowish or greenish grey, sometime white-pruinose; epithecium pale yellowish brown or brownish; hypothecium sometimes brownish; paraphyese branched, asci broadly clavate, short-stalked. ascospores (7-)8.5-14 $\times(3-) 4-7 \mu \mathrm{m}$.

Specimen Examined:-3: on Alkaloid feldspar granite rock, 2008, \# 2060.

\section{Lecanora garovaglii (Körb.) Zahlbr}

Thallus placodioid, $4-6 \mathrm{~cm}$ or more in diam, $0.5-2(3) \mathrm{mm}$ thick in centre, distinctly rosetted, the lobe tips and occasionally edges frequently dark olivaceous to bluish black; areoles contiguous, irregular; upper cortex without dead algal cells, $15-50 \mu \mathrm{m}$ thick; medulla whith, or darkening towards lower side, very loose, becoming hollow in center; lower surface pale grey and arachnoid, or towards edges and tips smooth and strong to dark grayish to yellowish brown or green to black; lowr cortex 8-18 $\mu \mathrm{m}$ thick, \pm continuous; apothecium scattered, to crowded towards thallus centre, asci narrowly clavate, 8 -spored; ascospores hyaline, simple, ellipsoid to broadly ellipsoid or ovoid-globose, $8-12 \times 5-6(7) \mu \mathrm{m}$.

Specimens Examined: -1 : on Alkaloid feldspar granite rock, 2007, \# 2061, 2081.

Lecanora muralis (Schreb.) Rabenh.

Thallus placodioid, rosettes, marginal lobes flat to concave; thallus centre sometimes areolate, greenish yellow to yellow brown, apothecium sessile, densely aggregated in the centre of the thallus; thalline exciple well developed, entire to crenulate or flexuose; disc yellow-brown to reddish brown, flat to slightly convex, not pruinose; epithecium pale yellowish or brownish; paraphyese mainly simple or sparsely branched above, apices not swollen or capitate; asci 30-40 × 8-12 $\mu \mathrm{m}$; ascospores $9-15(-16) \times(4-) 5-7 \mu \mathrm{m}$. 
Specimens Examined: -1: on Alkaloid feldspar granite rock, 2007, \# 2062, 2084

\section{**Lecidea tessellate Flörke}

Thallus crustose, usually well developed, \pm regulary areolate to rimose-areolate, whitish grey to pale bluish grey, esorediate; prothallus black, obvious at the margine of the thalli or indistinct or lacking; ascomata apothecia; apothecium black, singular or in sometimes large and dense groups; asci clavate, 8-spored; ascospores hyaline, simple, broadly ellipsoid to oblong- ellipsoid.

Specimen Examined: -3: on Alkaloid feldspar granite rock, 2008, \# 2063.

\section{Lecidella carpathica Körb.}

Thallus verrucose, usually well developed, white or pale to dark grey; apothecium immersed, at first flat, later becoming \pm convex; epithecium partly greenish black, brownish tinged; hypothecium semi-opaque, bright redbrown; ascospores 10-16 × 6-8.5 $\mu \mathrm{m}$.

Specimens Examined: -2, $1560 \mathrm{~m}$, on Alkaloid feldspar granite rock, 2007, \# 2064, 2083.

Psora decipiens (Hedw.) Hoffm.

Thallus squamulose, sometimes overlapping, adpressed, bright pink-red-brown, epruinose or partly whitepruinose, apothecium sessile, marginal, black, epruinose or \pm white-yellow- pruinose; ascospores $11-18 \times 6-8 \mu \mathrm{m}$. Specimen Examined: -2: Alkaloid feldspar granite rock, 2007, \# 2065.

\section{Rhizocarpon geminatum Körb.}

Thallus crustose; rounded, flat to convex gray areolate; on black prothallus which is visible among the areoles and sometimes at the margin of thallus. apothecium between or on thallus areoles; disc convex, black, epruinose. asci 2-spored; ascospores dark greenish brown, strongly muriform.

Specimens Examined: -3: Alkaloid feldspar granite rock, 2008, \# 2066.

\section{Rhizocarpon geographicum (L.) DC.}

Thallus crustose, with angular, flat to convex scattered areoles; on or among a conspicuous black prothallus that is also visible at the margin of the thallus; surface yellow-green or bright yellow. Apothecium between areoles; disc round or angular, flat to slightly convex, black, epruinose. asci clavate, 8-spored; ascospores (20-)22-40(46) $\times 10-19(-22) \mu \mathrm{m}$, dark greenish brown-black, moriform. Specimen Examined: -3: Alkaloid feldspar granite rock, 2008, \# 2067.

\section{Rhizocarpon viridiatrum (Wulfen) Körb.}

Thallus crustose; convex areolate; lichenicolous, surface matt, bright green; conspicuous prothallus. apothecium relatively large, on or between areoles; disc black, convex, rounded, epruinose; asci clavate, 8-spored; ascospores 12-24 × 7-13 $\mu \mathrm{m}$, dark brown, moriform.

Specimen Examined: -3: Alkaloid feldspar granite rock, 2008, \# 2068.
Rhizoplaca melanophthalma (Ramond) Leuckert \& poelt Thallus mostly $0.5-1.5(-2.5) \mathrm{cm}$ across, polyohyllous and often appearing squamulose or pulvinate; upper surface dull to shiny, occasionally \pm pruinose, usually light to moderate greenish yellow; lower surface blue-black near edges, usually continuous, smooth to uneven or roughened; ascomata apothecia; apotheciumimmersed then sessile; disc cocave to plane or undulate, yellowish brown to moderate brown, olive, or greenish to bluish black, epruinose or weakly to densely pruinose; ascospores \pm ellipsoid to subglobose but rather variable in size and shape.

Specimen Examined: -2: Alkaloid feldspar granite rock, 2007, \# 2069.

**Rhizoplaca peltata (Ramond) Leuckert \& poelt Thallus to $3 \mathrm{~cm}$ across, distinctly umbilicate, usually monophyllous but sometimes strongly lobed; upper surface \pm pale greenish yellow to yellow, continuous to strongly rimose, epruinose to partly pruinose, matt or slightly nitid, edges concolorous or blackened, sometime with thallospores; lower surface \pm yellowish brown, to bluish black near edges, smooth to uneven, \pm strongly cracked towards centre, with medulla showing through; apothecium usually common, to $2-3 \mathrm{~mm}$ diam, long remaining immersed to broadly adnate or at least adnate; disc concave then plane or sometimes convex, epruinose, orangish yellow to yellowish or reddish brown; ascospores ellipsoid to subglobose, $9-12 \times 5-8 \mu \mathrm{m}$.

Specimen Examined: -3 : Alkaloid feldspar granite rock, 2008, \# 2070.

\section{Toninia candida (Weber) Th. Fr.}

Thallus squamulose; rosulate, marginal squamules weakly concave to weakly convex, forming more or less radiating lobes; apothecium up to $2 \mathrm{~mm}$ diam, weakly concave to weakly convex, persistently marginate, densely pruinose; hypothecium medium brown to dark reddish brown in upper part, paler in lower part;hymenium 60-70 $\mu \mathrm{m}$ high; epithecium gery; ascospores fusueform, 1-septate, $15-24 \times 3-4 \mu \mathrm{m}$.

Specimen Examined: -3: Alkaloid feldspar granite rock, 2008, \# 2071.

Toninia diffracta (A. Massal.) Zahlbr.

Thallus squamulose; upper surface grey, densely whitepruinose or more rarely partly not pruinose; apothecium black, weakly concave to weakly convex;epithecium grey; hymenium colourless to pale brown; hypothecium lower part brown, upper part dark brown, ascospores 14-20 × 3-5 $\mu \mathrm{m}, 1$-septate, fusiform.

Specimen Examined: -3: Alkaloid feldspar granite rock, 2008, \# 2072.

**Verrucaria lecideoides Trevis.

Thallus cracked-areolate, gery to greyish brown; prothallus present or absent; algal layer 5-20 $\mu$ mthick; ascomata perithecia; perithecium 1-2 per areole; 
ascospores $5-9 \times 14-20 \mu \mathrm{m}$

Specimen Examined: -3 : Alkaloid feldspar granite rock, 2008, \# 2073.

Xanthoria elegans (Link.) Th. Fr.

Thallus to $4 \mathrm{~cm}$, forming \pm regular pale orange to reddish orange rosettes; lobes \pm uniformly $0.5-1 \mathrm{~mm}$ wide throughout their length, \pm nodulose, contiguous or \pm overlapping at centre, markely plicate, strongly convex; apothecium usually abundant throughout the central area of thallus, with persisten thalline exciple; ascospores (9) $11 \times 5-7(-9) \mu \mathrm{m}$, ellipsoid or ovoid.

Specimen Examined: -3: Alkaloid feldspar granite rock, 2008, \# 2075.

\section{REFERENCES}

Aghanabati, A. (2004). Geology of Iran, Geological Survey of Iran, $583 \mathrm{pp}$.

Awasthi, D. D. (1991). A Key to the Microlichens of India, Nepal and Sri Lanka. J. Carmer, Berlin. Stuttgart. 321 pp.

Haida, J. (2003). A Taxonomic study of the calcicolous endolitic species of the genus Verrucaria (Ascomycotina, Verrucariales) with the lid- like and radiately opening involucrellum. Act mus Richnov. Set. Nature, 10(1): 148 pp.

Magnusson, H. A., (1929). A Monograph of Genus Acarospora. Kongl. Svenska Vetenskapskad. Handl. Ser. 10. Pp 207208.

Mc Cune, B. (2002). Key to the lichen Genera of the pacific Northwest, Dept. Botany and Plant Pathology, Oregon State University, Corvallis, oregon USA. 83 pp.

Moniry, H. M., Fallahin, F. and Maassoumi, A. (2005). Lichens from Khorasan province, Iran. Folia Crytogamica Estonica, 41: 55-57.

Nash, T. H., Ryan, B. D., Gries. C. and Bungartz, F., (2002). Lichen Flora of The Greater Sonoran Desert Region. Vol I, Arizon Stata University, Tempe, Arizon, USA. 532 pp.
Nash, T. H., Ryan, B. D., Gries. C. and Bungartz, F. (2002). Lichen Flora of The Greater Sonoran Desert Region. Vol II, Arizon Stata University, Tempe, Arizon, USA. 742 pp.

Orange, A., W. James, P and J. White, F. (2001). Microchemical methods for the Identification of Lichens. British Lichen Society, UK.102 pp.

Oxner, A. N. (1971). Genus Aspicilia. - Pp. 146-217 in: Kopaczevskaja, E. G., Makarevicz, M. F., Oxner, A. N. and Rassadina, K. A. (eds.), Handbook of the lichens of the U.S.S.R. 1. Leningrad. [Translation by M. P. Zhurbenko, ca. 2004, translation financed and provided by Bjorn OweLarsson]

Purvis, W.o., Coppins, B. J., Hawksworth, D. L., James, P.W. and Moor, D.M., (1992). The Lichen Flora of Great Britain and Ireland. The British Lichen Society. $710 \mathrm{pp}$.

Rashed, M. H., Ayatollahi, M., Joharchi, M. R., Akbarzadeh, M. and Safavi, H. (1982-1987). Khorasan Vegetation, A Report on Plant Collection and Identification of Khorasan Province I, II. Ferdowsi University of Mashad, Iran.

Seaward M. R. D., Sipman, H. J. M., Schultz M., Maassoumi , A. A., Haji moniri Anbaran, M. and Sohrabi M. (2004). A preliminary lichen checklist for Iran. Willednowia 34: 543576.

Seaward M. R. D., Sipman H. J. M. and Sohrabi M. (2008). A revised checklist of lichenized. Lichencolouse and allied fungi Iran. Sauteria 15: 459-420.

Szatala, O. (1940). Lichenes.- [In: Rechinger, K. H., Baumgartner, J., Petrak, F. and Szatala, O., Ergebnisse einer botanischen Reise nach dem Iran]. Ann. Naturhist. Hofmus., 50: 521-533.

Szatala,O. (1957). Prodromus einer Flechtenflora des Irans.Ann. Hist.- Nat. Hung., ser. 2, 8: 101-154.

Timdal, E. (1991). Amonograph of the genus Toninia (lecideaceae, Ascomycetes). Opera Bot. 110: 1-137. 